Article

\title{
Symmetric Double-Eye Structure in Hurricane Bertha (2008) Imaged by SAR
}

\author{
Guosheng Zhang * (D) and William Perrie \\ Fisheries and Oceans Canada, Bedford Institute of Oceanography, Dartmouth, NS B2Y 4A2, Canada; \\ William.Perrie@dfo-mpo.gc.ca \\ * Correspondence: zgsheng001@gmail.com; Tel.: +1-902-426-7797
}

Received: 15 July 2018; Accepted: 13 August 2018; Published: 15 August 2018

\begin{abstract}
Internal dynamical processes play a critical role in hurricane intensity variability. However, our understanding of internal storm processes is less well established, partly because of fewer observations. In this study, we present an analysis of the hurricane double-eye structure imaged by the RADARSAT-2 cross-polarized synthetic aperture radar (SAR) over Hurricane Bertha (2008). SAR has the capability of hurricane monitoring because of the ocean surface roughness induced by surface wind stress. Recently, the C-band cross-polarized SAR measurements appear to be unsaturated for the high wind speeds, which makes SAR suitable for studies of the hurricane internal dynamic processes, including the double-eye structure. We retrieve the wind field of Hurricane Bertha (2008), and then extract the closest axisymmetric double-eye structure from the wind field using an idealized vortex model. Comparisons between the axisymmetric model extracted wind field and SAR observed winds demonstrate that the double-eye structure imaged by SAR is relatively axisymmetric. Associated with airborne measurements using a stepped-frequency microwave radiometer, we investigate the hurricane internal dynamic process related to the double-eye structure, which is known as the eyewall replacement cycle (ERC). The classic ERC theory was proposed by assuming an axisymmetric storm structure. The ERC internal dynamic process of Hurricane Bertha (2008) related to the symmetric double-eye structure here, which is consistent with the classic theory, is observed by SAR and aircraft.
\end{abstract}

Keywords: hurricane internal dynamical process; synthetic aperture radar (SAR); eyewall replacement cycles; ocean winds

\section{Introduction}

Accurate observations of surface winds of a tropical cyclone (hurricane or typhoon), particularly the high-resolution structures, play a critical role in improving hurricane dynamic readiness and understanding of its evolution process. Over the past 25 years, prediction skill for hurricane intensity has had comparatively few improvements because of limited knowledge regarding the hurricane internal dynamical processes, whereas its track forecasts errors have steadily declined [1]. Moreover, determinations of hurricane inner core structures and surface winds remain considerable operational challenges to the hurricane dynamic studies [2]. Routinely, surface winds of hurricanes are measured by the airborne Stepped Frequency Microwave Radiometer (SFMR) along flying track. Then, hurricanes are assumed to be axisymmetric. Based on this axisymmetric assumption, hurricane internal dynamics have been analyzed using the SFMR measurements, i.e., the vortex Rossby wave dynamics [3], eyewall replacement cycles (ERCs) [4], and hurricane pressure-wind model [5]. However, the actual hurricane structure, whether axisymmetric or not, is difficult to discern, only based on the aircraft reconnaissance low-level SFMR data. The surface wind fields of hurricanes have high azimuthal asymmetries, 
and these asymmetries are hard to measure by the aircraft, which typically flies at roughly fixed azimuths with time legs [5-7].

Compared with optical satellite sensors, the spaceborne synthetic aperture radar (SAR) is suitable for two-dimensional ocean surface wind field observations, for its advantages of a high spatial resolution, relatively large spatial swath, and its ability to work day-and-night under almost all-weather conditions. Additionally, hurricanes have been frequently observed by spaceborne SAR images, since the first spaceborne SAR image became available in 1978 [8]. Over the last few decades, SAR observations have been adopted in much research on hurricane readiness [9], morphology [8,10], precipitation [11], ocean surface current [12], and hurricane internal dynamic processes [13]. In this study, we present an analysis of one cross-polarized ScanSAR image from C-band RADARSAT-2 over Hurricane Bertha (2008) containing a double-eye structure.

To estimate the hurricane double-eye core structure from observed SAR surface winds, the idealized surface wind structure model, known as the Symmetric Hurricane Estimates for Wind (SHEW) model, is extended from a single-eye to double-eye structure. The double-eye SHEW model is the successor of approaches that we proposed in 2014 and 2017 [10,14]. To simplify the process, we present the SHEW model for the double-eye structure based on three assumptions: (1) both the hurricane eyewalls containing the maximum winds are circular-shaped; (2) the maximum wind speeds on either eyewall are axisymmetric; and (3) the wind speed within a hurricane is a function of the radius.

The theory for ERCs related to the hurricane double-eye structure was originally presented by Willoughby et al. (1982) [15]. This theory suggests that any secondary eyewall will shrink and contract due to the presence of annular convective heating. The circulation in a hurricane is changed by the axisymmetric outer eyewall heating, which results in cutting off the boundary layer inflow to the primary eyewall. Then, the hurricane is weakened by the outage of boundary layer inflow $[15,16]$. Based on an analysis of 79 Atlantic basin hurricanes observed by aircraft reconnaissance, Sitkowski et al. (2011) [4] demonstrated that the maximum wind speed of the primary core decreases significantly after the formation of the outer eyewall in a general ERC process. The RADARSAT-2 VH-polarized ScanSAR image over Hurricane Bertha (2008) provides us with a chance to study the sea wind field of a double-eye structure and its relationship to the ERC internal dynamic process.

The modified SHEW model for a double-eye structure is based on the one-dimensional modified Rankine vortex functions $[3,4,17]$ and double circles for the eyewalls containing maximum wind speeds. We organize the remainder of this paper as follows. The data sets of cross-polarized SAR observations from C-band RADARSAT-2 and the respective aircraft SFMR measurements, as well as the double-eye SHEW model, are presented in Section 2. Then, we show the extracted double-eye structure of Hurricane Bertha (2008) in the SHEW model in Section 3 and discuss the respective ERC process with the aircraft measurements in Section 4. Finally, we present conclusions in Section 5.

\section{Materials and Methods}

Bertha (2008) was an early season category 3 hurricane and the longest-lived Atlantic July tropical cyclone on record. In this study, a hurricane wind speed retrieval model is employed and an idealized vortex model for a symmetric double-eye hurricane structure is proposed.

\subsection{Data Sets}

To study the role of the symmetric secondary eyewall, we adopt one Cross-polarized (VH-polarized) ScanSAR image from C-band RADARSAT-2 in dual-polarized (VV and VH) mode (acquired at 10:14 UTC, 12 July 2008) over Hurricane Bertha (2008). To show the respective ERC process, the aircraft SFMR (stepped-frequency microwave radiometer) measurements acquired at times close to the SAR image are also employed. We show the SAR image, aircraft tracks, and the SAR location with respect to the Best Track of Hurricane Bertha (2008) in Figure 1. 
The VH-polarization SAR image from C-band RADARSAT-2 used here is in ScanSAR wide swath mode. Its medium resolution is $50 \mathrm{~m}$ and the swath width is about $450 \mathrm{~km}$. To reduce the image speckle noise, we calibrated the SAR image and then downscaled the spatial resolution to $1 \mathrm{~km}$ using the boxcar averaging method [18]. As shown in Figure 1, the SAR image here captured the whole core structure of Hurricane Bertha (2008). We retrieved the wind speeds directly from the SAR image using the C-3PO (C-band Cross-Polarization Coupled-Parameters Ocean) hurricane wind retrieval model, which was developed for cross-polarization SAR wind speed retrieval [19]. The operational surface winds measured by the SFMR radar on board the research aircrafts of NOAA (National Oceanic and Atmospheric Administration) WP-3D and U.S. Air Force are adopted. The SFMR radar can potentially provide along-track mapping of wind speeds at relatively high temporal $(1 \mathrm{~Hz})$ and spatial $(\sim 120 \mathrm{~m})$ resolutions. The root-mean-square errors of the SFMR winds are less than $4 \mathrm{~m} / \mathrm{s} \sim 5 \mathrm{~m} / \mathrm{s}[17,20]$, with validations of the measurements from dropwindsonde and an in situ instrument.

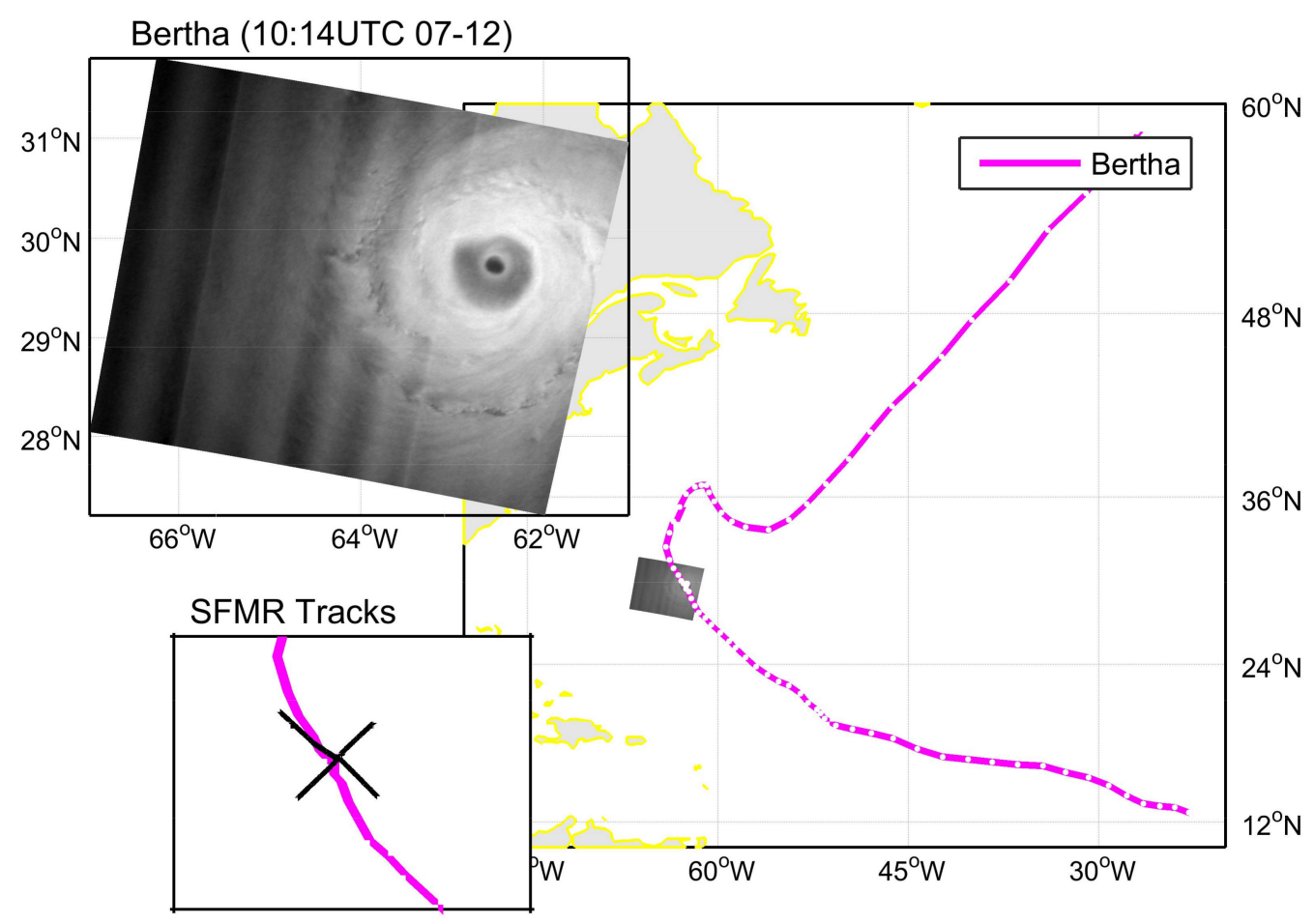

Figure 1. VH-polarized SAR image from C-band RADARSAT-2 over Hurricane Bertha (10:14 UTC, 12 July 2008), as well as flight tracks of SFMR measurements relative to the same hurricane internal dynamic processes and the Best Track (BT) of Hurricane Bertha (2008). RADARSAT-2 Data and Product MacDonald, Dettwiler, and Associates Ltd., All Rights Reserved.

\subsection{C-3PO Hurricane Wind Retrieval Model}

We retrieved the sea surface wind from the knowledge of normalized radar cross section (NRCS) imaged by SAR, using the C-3PO hurricane wind retrieval model. The C-3PO hurricane high-wind-speed retrieval model is [19]:

$$
\sigma_{0}[d B]=\left[0.2983 \cdot u_{10}-29.4708\right] \cdot\left[1+0.07 \cdot \frac{\theta_{\text {ind }}-34.5}{34.5}\right]
$$

where $u_{10}$ is the surface wind speed (at $10 \mathrm{~m}$ reference height), $\sigma_{0}$ is the NRCS of the radar signal with the units of $\mathrm{dB}$, and $\theta_{\text {ind }}$ is the SAR incidence angle.

The C-3PO model was developed from a theoretical analysis and a database including 650 sets of wind vectors, VH-polarized NRCSs, and the associated incidence angles [19]. This database was built 
based on five RADARSAT-2 VH-polarized SAR images covering five different hurricanes, as well as the collocated wind vectors measured by the aircraft SFMR. The SFMR wind vectors were selected during the 30-mintute windows with respect to the SAR acquired time. As the aircraft measured the rain rate simultaneously with the wind vector, we removed the measurements with rain rates higher than $10 \mathrm{~mm} / \mathrm{hr}$. Then, we collocated the VH-polarized NRCSs with the wind vectors by considering the hurricane movements and rotations.

The incidence angles should play a role in the cross-polarized (VH/HV) SAR wind retrieval. However, the dependences of the wind induced cross-polarized NRCSs on the incidence angles are difficult to extract because of a lack of observational data. Based on this limited database, we [19] developed a wind retrieval model (C-3PO), including the incidence angles, by a theoretical analysis. In fact, in that study, we proposed a theoretical model for the C-band Cross-polarization based on two existing theoretical models developed for the Co-polarizations (VV and $\mathrm{HH}$ ). We simulated a relationship between the VH-polarized NRCSs and the incidence angles for various wind speeds. We show the C-3PO hurricane wind retrieval model and the database in Figure 2.

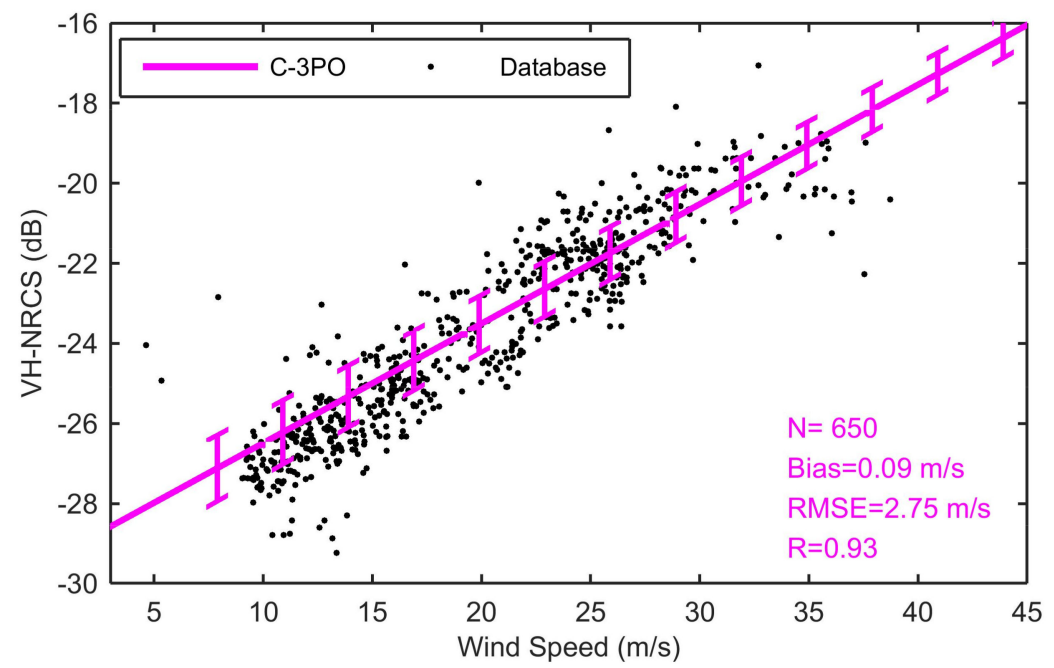

Figure 2. C-3PO hurricane wind retrieval model. The magenta line is for the middle incidence angle $\left(34.5^{\circ}\right)$, as well as the bars for the incidence angle ranges (from $19.5^{\circ}$ to $49.5^{\circ}$ ) of the RADARSAT-2 ScanSAR mode.

\subsection{SHEW Idealized Vortex model}

For the symmetric double-eye structure estimation, our SHEW model [10] is modified using the double modified Rankine vortex functions [4,17]. Using a given hurricane center (HE) location, we firstly transfer the geographical coordinates to a polar coordinate system in the modified SHEW model:

$$
\left\{\begin{array}{l}
r=\operatorname{distance}(\text { grid, } H E) \\
\theta=\operatorname{azimuth}(\text { grid }, H E)
\end{array}\right.
$$

In this model, $\theta=0$ means east and rises counter-clockwise. Then, we propose the symmetric double-eye SHEW model as: 


$$
u_{f}(r, \theta)=\left\{\begin{array}{lr}
u_{1}(\theta) \cdot\left[\frac{r}{r_{1}(\theta)}\right], & \left(r \leq r_{1}(\theta)\right) \\
u_{1}(\theta) \cdot\left[\frac{r_{1}(\theta)}{r}\right]^{\alpha_{1}}, & \left(r_{1}(\theta)<r \leq r_{\text {moat }}\right) \\
u_{1}(\theta) \cdot\left[\frac{r_{1}(\theta)}{r_{\text {moat }}}\right]^{\alpha_{1}} & \\
+\left\{u_{2}(\theta)-u_{1}(\theta) \cdot\left[\frac{r_{1}(\theta)}{r_{\text {moat }}}\right]^{\alpha_{1}}\right\} \cdot\left[\frac{r-r_{\text {moat }}}{r_{2}(\theta)-r_{\text {moat }}}\right], & \left(r_{\text {moat }}<r \leq r_{2}(\theta)\right) \\
u_{2}(\theta) \cdot\left[\frac{r_{2}(\theta)}{r}\right]^{\alpha_{2}}, & \left(r_{2}(\theta)<r \leq 150 \mathrm{~km}\right)
\end{array}\right.
$$

where $u_{1}(\theta)$ and $u_{2}(\theta)$ are the maximum wind speeds for the inner and outer vortexes, respectively; $r_{1}(\theta)$ and $r_{2}(\theta)$ are the radius of the maximum wind (RMW) for the inner and outer vortexes, respectively; and $\alpha_{1}$ and $\alpha_{2}$ are the decay parameters for the two vortexes, respectively. Moreover, a specific pivot position between the two vortexes is labeled $\left(u_{\text {moat }}, r_{\text {moat }}\right)$. For symmetric hurricane structures, the maximum wind speeds and RMWs are set as constants, $\left(u_{1}, u_{2}\right)$ and $\left(r_{1}, r_{2}\right)$, respectively. In Figure 3, we provide an example for the symmetric double-eye hurricane structure using the SHEW model with the following values of $\left(u_{1}=35 \mathrm{~m} / \mathrm{s}, r_{1}=15 \mathrm{~km}, \alpha_{1}=0.5\right)$ for the inner vortex, and $\left(u_{2}=35 \mathrm{~m} / \mathrm{s}\right.$, $\left.r_{2}=45 \mathrm{~km}, \alpha_{2}=0.5\right)$ for the outer vortex, as well as $\left(u_{\text {moat }}=25 \mathrm{~m} / \mathrm{s}, r_{\text {moat }}=33 \mathrm{~km}\right)$ for the pivot position.

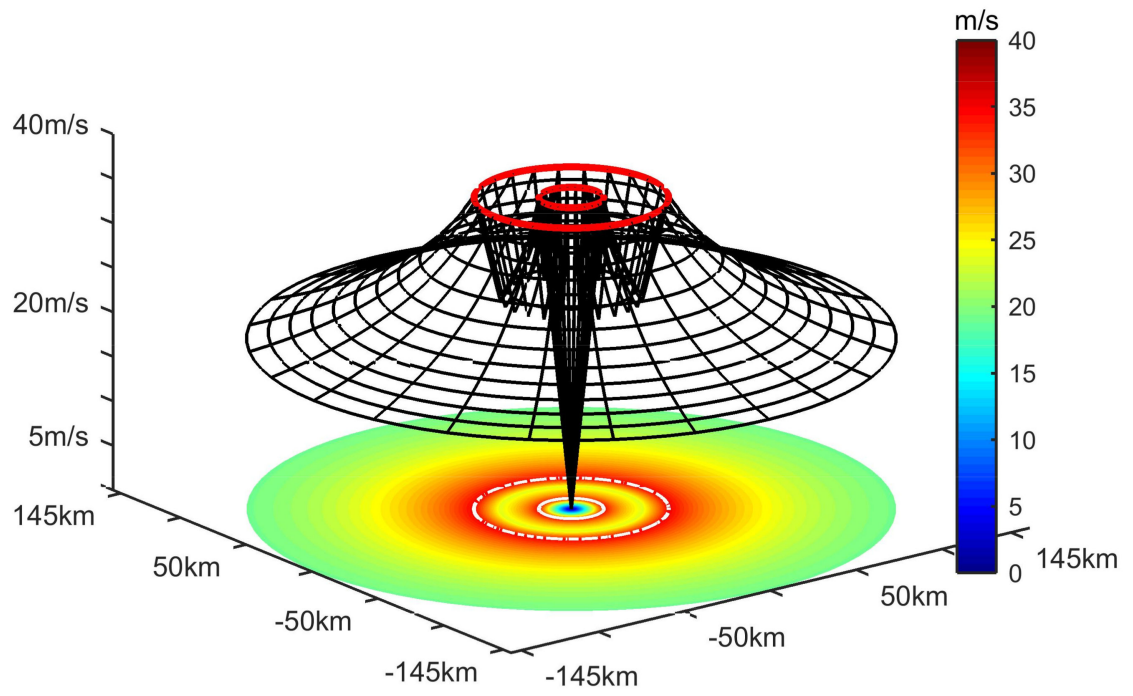

Figure 3. An example of the SHEW symmetric double-eye hurricane structure. The maxima wind speeds for two eyes are both $35 \mathrm{~m} / \mathrm{s}$, the RMW for the inner core is $15 \mathrm{~km}$ and for the secondary core is $45 \mathrm{~km}$, and the two decay parameters are both 0.5 .

To estimate the double-eye structure of Hurricane Bertha (2008), we display the procedures in the flowchart (Figure 4) needed to use the modified SHEW. Firstly, we give an initial hurricane center to start the procedures. With various parameters, we fit the winds relative to the radius from the SHEW model to the SAR winds using the least-squares approximation. We note that the parameters of the SHEW model are independent. 


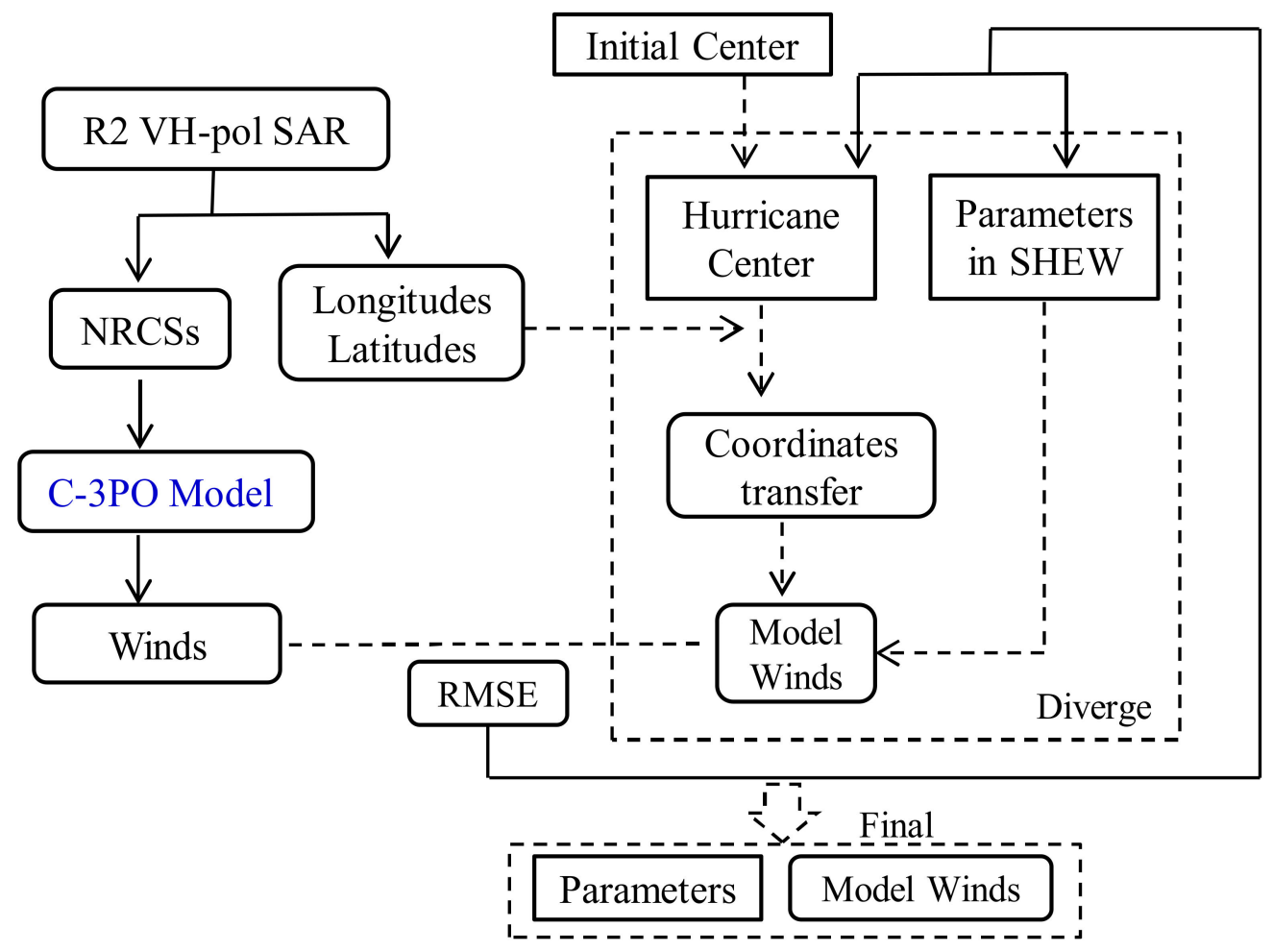

Figure 4. Flowchart for the modified SHEW model to estimate the hurricane double-eye structure.

\section{Results}

In Figure 5a, we show the time-series of hurricane Bertha's intensity from the Best Track database and the corresponding SAR image. In Figure 5b, we show the wind fields retrieved from the VH-polarized SAR image by employing the C-3PO model (Equation (1)). Relative to the hurricane center, identified by very low wind speeds, two high wind speed rings are obviously separated by relatively low wind areas. Following the flowchart in Figure 4, we estimate the closest idealized axisymmetric hurricane structure, as shown in Figure 5c, by fitting the symmetric double-eye SHEW model (Equations (2) and (3)) to wind field retrieved from SAR. Although the maximum wind speed of the inner eyewall is much smaller than the outer eyewall, the outline of the inner core is very clear in the wind speed field. The detected intensity of the inner core is $20.9 \mathrm{~m} / \mathrm{s}$ and for the secondary eyewall, $27.9 \mathrm{~m} / \mathrm{s}$. The RMW for the primary vortex is $13 \mathrm{~km}$ and for the secondary vortex, $52 \mathrm{~km}$. Within the estimated axisymmetric framework (Figure 5c), we show the storm-centered wind field of Hurricane Bertha (2008) observed by SAR in Figure 5d. Using the wind fields reconstructed by the modified SHEW model (Figure 5c) and imaged by SAR (Figure 5d), we compute the standard deviation between the closest idealized axisymmetric structure and the 'real' wind field as $2.45 \mathrm{~m} / \mathrm{s}$, as well as the correlation coefficient as 0.827 . The confidence interval of the correlation coefficient is higher than 99\%. In a related hurricane study, Zhang et al. (2017) [10] demonstrated that correlation coefficients between the idealized structure by the SHEW model and 'real' wind imaged by SAR are routinely between 0.60 to 0.85 . By comparison, the correlation coefficient here of 0.827 implies that the double-eye structure of Hurricane Bertha (2008) observed by SAR is close to symmetric. 
(a) Hurricane Bertha 3-20 July 2008
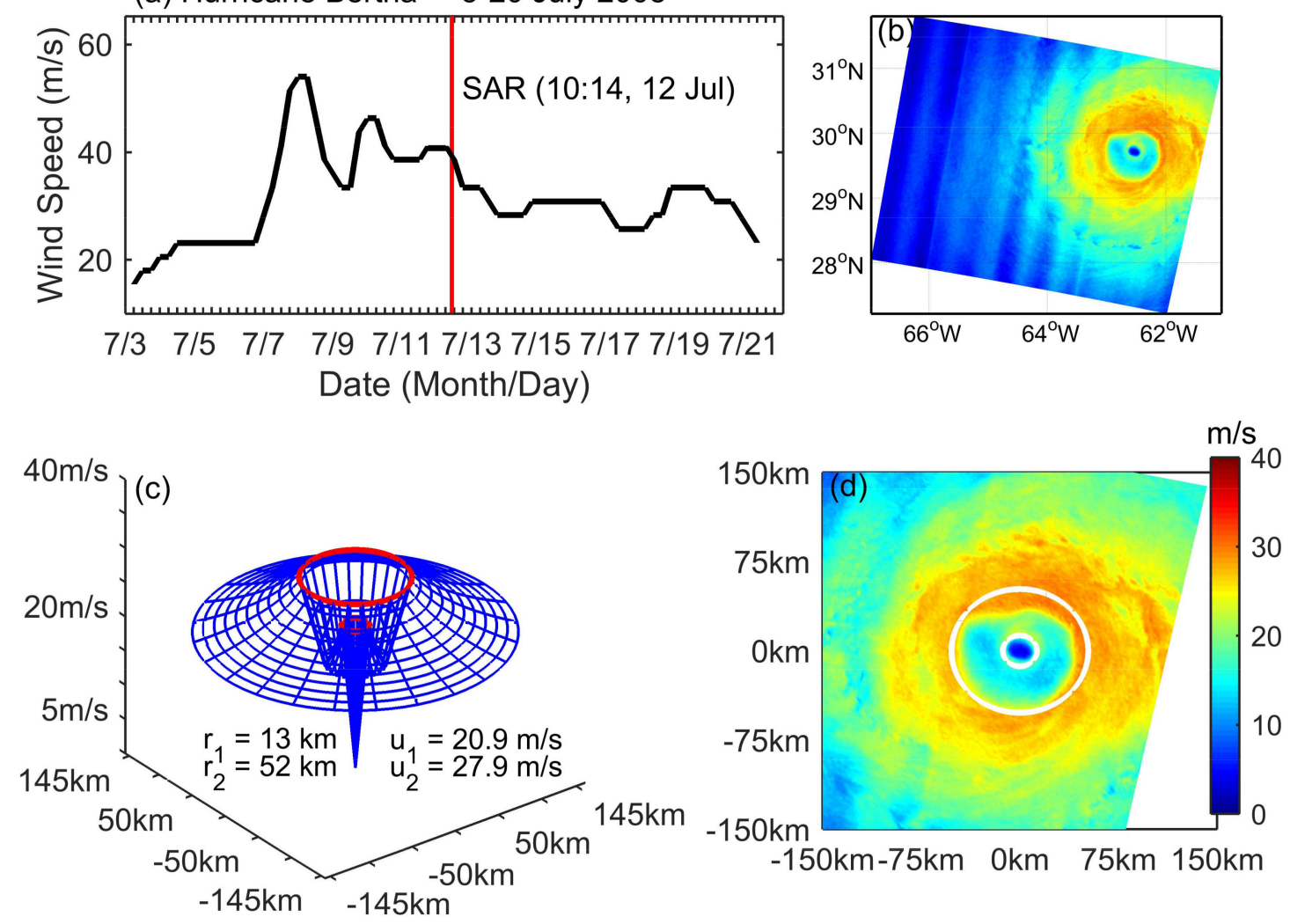

Figure 5. Hurricane Bertha (2008): (a) SAR captured time respective to the time series of Best Track Intensities; (b) retrieved sea surface wind speed from SAR images acquired at 10:14 UTC, 12 July 2008; (c) SHEW estimated closest idealized structure; and (d) storm-centered wind field of hurricane core.

In the axisymmetric framework for storm internal dynamic processes, Willoughby et al. (1982) [15] suggested that the hurricane intensity decreases as a result of the outage of boundary layer inflow to the primary eyewall, which is cut off by a negative tendency induced by the secondary eyewall heating. According to the classic ERC theory, the symmetric double-eye structure of Hurricane Bertha (2008) observed by SAR suggests that the secondary eyewall would contract and replace the primary eyewall.

As the SAR imagery of hurricane Bertha (2008) captures a double-eye structure (Figure 5), we address two points consistent with the classic theories for the ERC internal dynamic process: (1) the structure of the sea surface wind field is axisymmetric, and (2) the intensity of the inner eyewall is smaller than the outer eyewall.

\section{Discussion}

Routinely, tangential wind profiles are used for the aircraft measurements in applications for studies on the hurricane internal dynamic processes [14]. We adopt the four profiles associated with the SAR image which should be seen during one ERC process. Based on an analysis of 79 Atlantic basin hurricanes observed by aircraft reconnaissance, Sitkowski et al. (2011) [4] demonstrated that the process of replacing an inner eyewall with an outer eyewall lasts for an average of $36 \mathrm{~h}$, in a general ERC process. They [4] also suggested that the maximum wind speed of the primary core decreases significantly after the formation of the outer eyewall. The maximum difference between the time of SAR observation and SFMR winds used here is no more than $10 \mathrm{~h}$. Therefore, they are supposed to be part of the same ERC process.

To analyze the axisymmetric double-eye structure, we adopt the definition of the complete profile [13], which contains two continuous normal profiles across the hurricane center (one is flying 
into the hurricane and the other is flying out). As shown in Figure 6b, there are two complete profiles composited by four normal profiles. Although only four normal profiles were measured by SFMR, these measurements allow us to present a clear display of the hurricane evolution with the axisymmetric double-eye structure in Figure 6. In Figure 6a, we show a time-series composed of intensities for the two eyewalls observed by spaceborne SAR and airborne SFMR. The hurricane intensities from SAR are derived from the SHEW model, and the intensities of SFMR are extracted from the profiles using the maximum wind speeds. An outline of the ERC internal dynamic process of Hurricane Bertha (2008) is shown by the two complete profiles with the axisymmetric double-eye structure (Figure 6b-d). In Figure 6b, we show the distributions of SFMR wind speeds along the flight tracks.

In Figure 6c, we show a complete wind profile, which was captured about seven hours after the SAR image (hereafter "the $7 \mathrm{~h}$ profile"). Additionally, "the $7 \mathrm{~h}$ profile" is the first SFMR measurement of the same secondary eyewall as that detected from the ScanSAR imagery. By comparing the storm-centered SAR wind field (Figure 5d) to the aircraft flying tracks (Figure 6b) of "the $7 \mathrm{~h}$ profile", the double-eye structure measured by SFMR is also axisymmetric, which indicates that this structure lasts more than seven hours. In Figure $6 \mathrm{~d}$, the other complete profile was measured about $8.5 \mathrm{~h}$ after the SAR image (hereafter "the $8.5 \mathrm{~h}$ profile"), which should be related to the same ERC process. We cannot distinguish the secondary eyewall in "the $8.5 \mathrm{~h}$ profile", which demonstrates that the inner eyewall was replaced by the outer eyewall during the period from $7 \mathrm{~h}$ to $8.5 \mathrm{~h}$ after the SAR image.
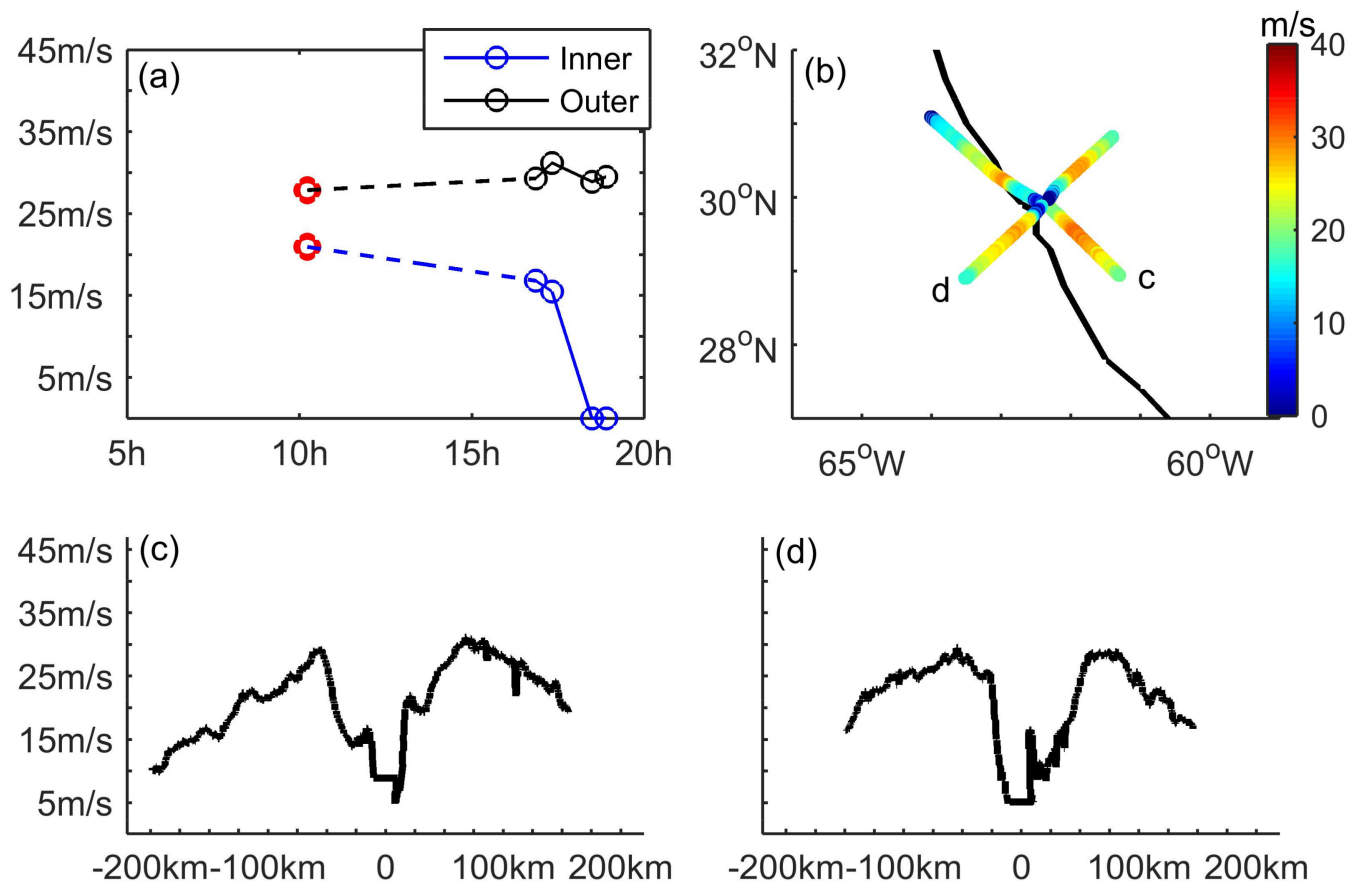

Figure 6. ERC dynamic process of Hurricane Bertha measured by aircraft SFMR: (a) hurricane intensities of the double-eye structure, (b) flight tracks, as well as (c), (d) the respective complete profiles along radius.

Recent studies [21] have suggested that symmetric heating appears to be the dominant factor for hurricane intensity change, although asymmetries controlled by the large-scale shearing environment may also be important. As is typical of hurricane processes during the axisymmetric framework, an early study of [16] demonstrated that a negative tendency for the tangential winds inside the secondary eyewall can be induced by the symmetric eyewall heating, which cuts off the boundary layer inflow to the primary eyewall and further weakens the hurricane intensity. Finally, towards the end of this progression, the primary eyewall was replaced by the secondary eyewall. Using two-dimensional observations of SAR with a high spatial resolution, one can see that the double-eye structure of 
Hurricane Bertha (2008) is relatively axisymmetric. Associated with the airborne SFMR measurements, we provide the relative ERC process. Therefore, the role of the axisymmetric double-eye structure in the ERC dynamic process is verified as consistent with the classic theory $[15,16]$. Moreover, many hurricanes are detected not to significantly weaken after the formation of the secondary eyewall [22], which may be a result of the asymmetric hurricane structure [13]. As Hurricane Bertha (2008) is relatively axisymmetric, the SFMR observed in one-dimension is supposed to represent the surface wind.

\section{Conclusions}

A one cross-polarized (VH) ScanSAR image from C-band RADARSAT-2 over Hurricane Bertha (2008) provides us with a chance to research the axisymmetric double-eye structure effects on the hurricane intensity change. In this study, we employ the C-3PO hurricane wind retrieval model and modify the SHEW idealized vortex model for the double-eye structure. By comparing the wind fields simulated by the symmetric double-eye SHEW model and observed by SAR, we compute the correlation coefficient of 0.827 with a confidence interval higher than $99 \%$ and the standard deviation of $2.45 \mathrm{~m} / \mathrm{s}$. In a related hurricane study, Zhang et al. (2017) [10] demonstrated that correlation coefficients between the idealized structure by the SHEW model and 'real' wind imaged by SAR are routinely between 0.60 to 0.85 , which implies that the double-eye structure of Hurricane Bertha (2008) observed by SAR is symmetric. To analyze the ERC process related to the double-eye structure captured by SAR, we employ two complete tangential wind profiles measured by airborne SFMR. After about $7 \mathrm{~h}$ from the SAR observation, the profile appears to capture the double-eye structure; but $8.5 \mathrm{~h}$ later, the primary eyewall should be replaced by the secondary eyewall.

Compared to the previous studies $[4,15,16,22]$, we find three characteristics consistent with the classic ERC theory as follows: (1) the structure of the sea surface wind field is axisymmetric; (2) the intensity of the primary eyewall is smaller than the secondary eyewall; and (3) the primary eyewall is replaced by the secondary eyewall. We suggest that the axisymmetric structure plays a major role in the ERC dynamic process.

Author Contributions: G.Z. was responsible for designing this study, processing datasets, building the model, and writing-Original Draft Preparation; W.P. was responsible for supervision, writing-review, and editing.

Funding: This research was funded by National Natural Science Youth Foundation of China under Grant 41706193, in part by the Canadian Space Agency SWOT and Office of Energy Research and Development (OERD) Programs, in part by the Canadian Data Utilization and Application Program (DUAP) "Winds from SAR" RCM Readiness Project between ECCC and DFO, and in part by the Open Fund of Key Laboratory of Geographic Information Science (Ministry of Education), East China Normal University (Grant No. KLGIS2017A06).

Acknowledgments: The authors thank the Canadian Space Agency for providing RADARSAT-2 data, NOAA HRD for supplying SFMR data (http:/ / www.aoml.noaa.gov/hrd/), and NOAA NHC for hurricane best track data (HURDAT2) (http:/ / www.nhc.noaa.gov/data/\#hurdat).

Conflicts of Interest: The authors declare no conflict of interest.

\section{References}

1. Montgomery, M.T.; Smith, R.K. Recent developments in the fluid dynamics of tropical cyclones. Annu. Rev. Fluid Mech. 2017, 49, 541-574. [CrossRef]

2. Sanabia, E.R.; Barrett, B.S.; Celone, N.P.; Cornelius, Z.D. Satellite and aircraft observations of the eyewall replacement cycle in Typhoon Sinlaku (2008). Monthly Weather Rev. 2015, 143, 3406-3420. [CrossRef]

3. Mallen, K.J.; Montgomery, M.T.; Wang, B. Reexamining the near-core radial structure of the tropical cyclone primary circulation: Implications for vortex resiliency. J. Atmos. Sci. 2005, 62, 408-425. [CrossRef]

4. Sitkowski, M.; Kossin, J.P.; Rozoff, C.M. Intensity and structure changes during hurricane eyewall replacement cycles. Mon. Weather Rev. 2011, 139, 3829-3847. [CrossRef]

5. Kossin, J.P. Hurricane wind-pressure relationship and eyewall replacement cycles. Weather Forecast. 2015, 30, 177-181. [CrossRef] 
6. Kossin, J.P.; Eastin, M.D. Two distinct regimes in the kinematic and thermodynamic structure of the hurricane eye and eyewall. J. Atmos. Sci. 2001, 58, 1079-1090. [CrossRef]

7. Franklin, J.L.; Black, M.L.; Valde, K. GPS dropwindsonde wind profiles in hurricanes and their operational implications. Weather Forecast. 2003, 18, 32-44. [CrossRef]

8. Li, X.; Zhang, J.A.; Yang, X.; Pichel, W.G.; DeMaria, M.; Long, D.; Li, Z. Tropical cyclone morphology from spaceborne synthetic aperture radar. Bull. Am. Meteorol. Soc. 2013, 94, 215-230. [CrossRef]

9. Du, Y.; Vachon, P.W. Characterization of hurricane eyes in RADARSAT-1 images with wavelet analysis. Can. J. Remote Sens. 2003, 29, 491-498. [CrossRef]

10. Zhang, G.; Perrie, W.; Li, X.; Zhang, J.A. A hurricane morphology and sea surface wind vector estimation model based on C-band cross-polarization SAR imagery. IEEE Trans. Geosci. Remote Sens. 2017, 55, 1743-1751. [CrossRef]

11. Zhang, G.; Li, X.; Perrie, W.; Zhang, B.; Wang, L. Rain effects on the hurricane observations over the ocean by C-band Synthetic Aperture Radar. J. Geophys. Res. 2016, 121, 14-26. [CrossRef]

12. Zhang, G.; Perrie, W. Dual-Polarized Backscatter Features of Surface Currents in the Open Ocean during Typhoon Lan (2017). Remote Sens. 2018, 10, 875. [CrossRef]

13. Zhang, G.; Perrie, W. Effects of asymmetric secondary eyewall on tropical cyclone evolution in Hurricane Ike (2008). Geophys. Res. Lett. 2018, 45, 1676-1683. [CrossRef]

14. Zhang, G.; Zhang, B.; Perrie, W.; Xu, Q.; He, Y. A hurricane tangential wind profile estimation method for C-band cross-polarization SAR. IEEE Trans. Geosci. Remote Sens. 2014, 52, 7186-7194. [CrossRef]

15. Willoughby, H.E.; Clos, J.A.; Shoreibah, M.G. Concentric eye walls, secondary wind maxima, and the evolution of the hurricane vortex. J. Atmos. Sci. 1982, 39, 395-411. [CrossRef]

16. Shapiro, L.J.; Willoughby, H.E. The response of balanced hurricanes to local sources of heat and momentum. J. Atmos. Sci. 1982, 3, 378-394. [CrossRef]

17. Wood, V.T.; White, L.W.; Willoughby, H.E.; Jorgensen, D.P. A new parametric tropical cyclone tangential wind profile model. Mon. Weather Rev. 2013, 141, 1884-1909. [CrossRef]

18. Shen, H.; Perrie, W.; He, Y.; Liu, G. Wind speed retrieval from VH dual-polarization RADARSAT-2 SAR images. IEEE Trans. Geosci. Remote Sens. 2014, 52, 5820-5826. [CrossRef]

19. Zhang, G.; Li, X.; Perrie, W.; Hwang, P.A.; Zhang, B.; Yang, X. A hurricane wind speed retrieval model for C-band RADARSAT-2 cross-polarization ScanSAR images. IEEE Trans. Geosci. Remote Sens. 2017, 55, 4766-4774. [CrossRef]

20. Horstmann, J.; Falchetti, S.; Wackerman, C.; Maresca, S.; Caruso, M.J.; Graber, H.C. Tropical cyclone winds retrieved from C-band cross-polarized synthetic aperture radar. IEEE Trans. Geosci. Remote Sens. 2015, 53, 2887-2898. [CrossRef]

21. Cotto, A.; Gonzalez, I., III; Willoughby, H.E. Synthesis of vortex Rossby waves. Part I: Episodically forced waves in the inner waveguide. J. Atmos. Sci. 2015, 72, 3940-3957. [CrossRef]

22. Kuo, H.-C.; Chang, C.-P.; Yang, Y.-T.; Jiang, H.-J. Western North Pacific typhoons with concentric eyewalls. Mon. Weather Rev. 2009, 137, 3758-3770. [CrossRef]

(c) 2018 by the authors. Licensee MDPI, Basel, Switzerland. This article is an open access article distributed under the terms and conditions of the Creative Commons Attribution (CC BY) license (http://creativecommons.org/licenses/by/4.0/). 